\title{
A Robust Skin Colour Segmentation Using Bivariate Pearson Type II $a \alpha$ (Bivariate Beta) Mixture Model
}

\author{
B.N.Jagadesh \\ Department of Computer Science and Engineering \\ Srinivasa Institute of Engineering \& Technology \\ NH-216, Cheyyeru, Amalapuram, A.P., INDIA \\ nagajagadesh@gmail.com \\ K.Srinivasa Rao \\ Department of Statistics \\ Andhra University \\ Visakhapatnam, A.P., INDIA. \\ ksraoau@yahoo.co.in \\ Ch.Satyanarayana \\ Department of Computer Science and Engineering \\ Jawaharlal Nehru Technological University Kakinada \\ Kakinada, A.P., INDIA. \\ chsatyanarayana@yahoo.com
}

\begin{abstract}
Probability distributions formulate the basic framework for developing several segmentation algorithms. Among the various segmentation algorithms, skin colour segmentation is one of the most important algorithms for human computer interaction. Due to various random factors influencing the colour space, there does not exist a unique algorithm which serve the purpose of all images. In this paper a novel and new skin colour segmentation algorithms is proposed based on bivariate Pearson type II $a \alpha$ mixture model since the hue and saturation values always lies between 0 and 1 . The bivariate feature vector of the human image is to be modeled with a Pearson type II $a \alpha$ mixture (bivariate Beta mixture) model. Using the EM Algorithm the model parameters are estimated. The segmentation algorithm is developed under Bayesian frame. Through experimentation the proposed skin colour segmentation algorithm performs better with respect to segmentation quality metrics such as PRI, VOI and GCE. The ROC curves plotted for the system also revealed that the proposed algorithm can segment the skin colour more effectively than the algorithm with Gaussian mixture model for some images.
\end{abstract}

Index Terms - Bivariate Pearson type II $a \alpha$ mixture model, skin colour segmentation, HSI colour space, segmentation quality metrics

\section{INTRODUCTION}

The skin colour segmentation has gained lot of importance due to its diversified applications in content based image retrievals, face detection, face tracking, gesture analysis, human computer interaction, computer vision etc., In all these areas it makes use of machine recognition of skin. The computer recognition is complex and difficult because of its limitations and static knowledge which are different from human endeavor. For efficient and effective design of skin colour segmentation algorithms it is needed to utilize statistical modeling [1].

The skin colour segmentation is a part of clustering and classification problem with respect to pattern recognition methodologies. Modeling the skin colour of humanbeings is very challenging task in image analysis since it is influenced by various factors such as illumination, camera characteristics, ethnicity, individual characteristics, subject appearance, background colour, shadow and motion etc., [2][3].

Another important difficulty in skin colour segmentation is that, the colour image senses work based on colour mosaic, which is assortment of different spectral filters [4]. Hence in skin colour segmentation the colour space plays a dominant role for feature extraction. In colour image it is customary to consider the RGB, Normalized RGB and CIE-xyz [5 - 9]. The perceptual colour space like TSL, HSL, HSV and HSI are developed for capturing the colour features more effectively [10 - 14]. To utilize the concept of orthogonal (independence of each other) colour spaces such as YCbCr, YIQ, YUV, and YES are developed [15 - 20]. The other colour spaces like CIE-Lab, CIE-Luv are also utilized extensively in colour image processing and analysis [21] [7] [22]. Among all these colour 
spaces the HSI colour space has certain advantages since it separate channels and outline certain colour properties which are close to the visual conjunctive system of human being [23] [24]. In HSI colour space the hue and saturation values are correlated through the intensity. That is the hue and saturation values of the pixel are the functions of pixel intensity. Hence recently in colour image processing the HS bivariate feature vector is utilized [25].

Recently much work has been reported in literature regarding skin colour segmentation. Cai and Goshtasb [26] have studied a method for detecting human faces in colour images using the skin separation. L.M. Bergassa et al. [8] have developed and analyzed an unsupervised and adaptive Gaussian skin colour model. Hayit Greenspan et al. [27] have considered the mixture of Gaussian distributions for modeling the space colour and segmentation. Kakhamanu et al. [28] have reviewed the methods for skin colour modeling and detection. Chang-Yul Kim et al [3] studied a practical system for detecting obscene videos. Ki-won Byun and $\mathrm{Ki}-\mathrm{Gon}$ Nam [29] studied the skin region detection using mean shift algorithm based on the histogram approximation. Among all segmentation algorithms the model based segmentation methods are more efficient than other methods since they capture the local and global information of the images more effectively [30].

In model based skin colour segmentation it is customary to assume that the feature vector associated with the colour image is either Gaussian or Gaussian mixture model. But the Gaussian or Gaussian mixture model have certain drawbacks like the feature vector in each region are mesokurtic and having infinite range. In reality the feature vector in each region of the image (skin and non-skin regions) may not be mesokurtic and more so having finite range. To overcome this drawback in colour image segmentation based on Gaussian mixture model recently K.Srinivasarao et al [25] have developed the skin colour segmentation based on bivariate type $1 \mathrm{Va}$ mixture model. They assumed that the hue and saturation values of the skin and nonskin region follows Pearson type $1 \mathrm{Va}$ probability distributions with different parameters. The Pearson type $1 \mathrm{Va}$ is having the range from 0 to $\infty$ and also the bivariate distribution they consider is having a limitation of $0<x<y$ where $x$ and $y$ are the values of hue and saturation.

But in general the hue and saturation values are having the range from 0 to 1 and they can not be constrained with the condition like $\mathrm{x}<\mathrm{y}$ ( $\mathrm{x}$ and $\mathrm{y}$ are hue and saturation values). To model the feature vector of the regions (skin and non-skin) it is reasonable to consider the bivariate Pearson type II $a \alpha$ model. Hence the whole image can be characterized by bivariate type II $a \alpha$ Pearson mixture model. The bivariate Pearson type II $a \alpha$ is having the marginal probability density for hue and saturation values as beta distributions which are having the range 0 to 1 . They also include several types of frequency curves for different values of the parameters. This distribution also characterizes the negative correlation between the hue and saturation values of the image region. With this motivation, in this paper a skin colour segmentation algorithm is developed and analyzed by assuming the feature vector of the entire image following a bivariate Pearson type II $a \alpha$ mixture distribution.

The rest of the paper is organized as follows: section 2 given brief discussion about bivariate Pearson type II $a \alpha$ mixture model and its properties. Section 3 deals with the estimation of the model parameters using EM Algorithm. EM Algorithm is most efficient algorithm for estimating the model parameters in mixture distribution. Section 4 is to initialize the model parameters using moment method of estimation and Kmeans algorithm. In Section 5 the skin colour segmentation algorithm is presented based on likelihood function under Bayesian frame work. In section 6 the experimentation and performance evaluation of the proposed algorithm are discussed. The experimentation is carried with five face images take from JNTUK face database. The performance of the proposed algorithm is studied by computing the segmentation quality metrics like PRI, GCE and VOI. The efficiency of proposed algorithm is also studied through confusion matrix and ROC curves. Section 7 deals with conclusion and scope for further work in this area of research.

\section{BIVARIATE PEARSON TYPE II $a \alpha$ MIXTURE MODEL}

In skin colour analysis the classification of the image is done into two categories namely, skin and non-skin colour regions. The skin colour is different from the colour of most other natural objects in the world. To build the statistical model for the pixels in the image, the feature vector is extracted using colour spaces. In skin colour segmentation one has to use the chrominance component in extracting the features. Accordingly the hue and saturation under HSI colour space are used for skin colour detection. The statistical observations of hue and saturation which form a bivariate feature vector match closely with the bivariate Pearson type II $a \alpha$ distributions. The bivariate Pearson type II $a \alpha$ given by Kotz et al [31], is having non negative and asymmetric nature of the random variable. It also includes a wide variety of bivariate probability distributions. Here it is assumed that the feature vector of the pixel in skin or non-skin regions in the image follows a bivariate Pearson type II $a \alpha$ distribution. The Joint Probability density function of the feature vector is

$$
f(x, y / \theta)=\frac{\Gamma(m+n+p)}{\Gamma(m) \Gamma(n) \Gamma(p)} x^{m-1} y^{n-1}(1-x-y)^{p-1}
$$

$m, n, p>0$

$x, y>0$ and $x+y \leq 1$ 
$\theta$ is the parametric set such that $\theta=(m, n, p)$, $\mathrm{x}$ denote the hue value and $\mathrm{y}$ denote the saturation value of the pixel in the image.

The marginal probability density function of the hue value is

$$
f(x)=\operatorname{Beta}(m, n+p)
$$

Its mean is

$$
\frac{m}{m+n+p}
$$

Its variance is

$\frac{m(n+p)}{(m+n+p+1)(m+n+p)^{2}}$

The marginal probability density distribution of the saturation value is

$$
f(y)=\operatorname{Beta}(n, m+p)
$$

Its mean is

$$
\frac{n}{m+n+p}
$$

Its variance is

$$
\frac{n(m+p)}{(m+n+p+1)(m+n+p)^{2}}
$$

The Covariance between hue and saturation is

$$
\frac{-m n}{(m+n+p+1)(m+n+p)^{2}}
$$

The correlation coefficient between hue and saturation is

$$
-\sqrt{\frac{m n}{(n+p)(m+p)}}
$$

Since the entire image is a collection of skin and nonskin pixel regions which are characterized by a bivariate Pearson type II $a \alpha$ distribution, the feature vector associated with the whole image is modeled as a two component bivariate Pearson type II $a \alpha$ mixture model. Its Joint probability density function is

$$
h(x, y)=\sum_{i=1}^{2} \alpha_{i} f_{i}\left(x, y / \theta_{i}\right)
$$

Where, $0<\alpha_{i}<1$ and $\alpha_{1}+\alpha_{2}=1$ and $f_{i}(x, y)$ is as given equation (1).

\section{ESTIMATION OF THE MODEL PARAMETERS BY EXPECTATION MAXIMIZATION ALGORITHM}

The likelihood function of sample bivariate observations $\left(x_{1} y_{1}\right),\left(x_{2}, y_{2}\right),\left(x_{3}, y_{3}\right), \ldots,\left(x_{N}, y_{N}\right)$ drawn from an image with probability density function $h(x, y ; \theta)=\sum_{i=1}^{K} \alpha_{i} f_{i}\left(x_{s}, y_{s} ; \theta\right)$ where $f_{i}$ is the probability density function of a Pearson type II mixture distribution and is given by

$$
\begin{aligned}
\boldsymbol{L}(\boldsymbol{\theta}) & =\prod_{s=1}^{N}\left(\sum_{i=1}^{K} \alpha_{i} f_{i}\left(x_{s}, y_{s} ; \boldsymbol{\theta}\right)\right) \\
& =\prod_{s=1}^{N}\left(\sum_{i=1}^{K} \alpha_{i} \frac{\Gamma(m+n+p)}{\Gamma(m) \Gamma(n) \Gamma(p)} x^{m-1} y^{n-1}(1-x-y)^{p-1}\right)
\end{aligned}
$$

This implies that

$$
\begin{aligned}
\log L(\theta) & =\log \prod_{s=1}^{N}\left(\sum_{i=1}^{K} \alpha_{i} f_{i}\left(x_{s}, y_{s} ; \theta\right)\right) \\
& =\sum_{s=1}^{N} \log \left(\sum_{i=1}^{K} \alpha_{i} f_{i}\left(x_{s}, y_{s} ; \theta\right)\right)
\end{aligned}
$$

The model parameters are estimated by using the Expectation Maximization Algorithm (E.M Algorithm). The updated equation of the parameter $\alpha_{k}$ is

$$
\begin{aligned}
\alpha_{k}^{(l+1)} & =\frac{1}{N} \sum_{s=1}^{N}\left[t_{k}\left(x_{s,} y_{s} ; \theta^{(l)}\right)\right] \text { for } \mathrm{K}=1,2 . \\
& =\frac{1}{N} \sum_{s=1}^{N}\left[\frac{\alpha_{k}^{l} f_{k}\left(x_{s,} y_{s} ; \theta^{(l)}\right)}{\sum_{i=1}^{2} \alpha_{i}^{l} f_{i}\left(x_{s} y_{s} ; \theta^{(l)}\right)}\right]
\end{aligned}
$$

where, $f_{k}\left(x_{s,} y_{s} ; \theta^{(l)}\right)$ is as given equation (1).

The updated equation of $m_{k}$ at $(l+1)^{\text {th }}$ iteration is

$$
\begin{aligned}
& \sum_{s=1}^{N} t_{k}\left(x_{s}, y_{s} ; \theta^{(l)}\right) \log \left(x_{s}\right)+\sum_{s=1}^{N} t_{k}\left(x_{s}, y_{s} ; \theta^{(l)}\right) \frac{1}{\Gamma\left(m_{k}+n_{k}+p_{k}\right)}\left(-\Gamma\left(m_{k}+n_{k}+p_{k}\right)\right. \\
& \left.* \log (\log (e))+\Gamma\left(m_{k}+n_{k}+p_{k}\right) * \psi\left(m_{k}+n_{k}+p_{k}\right)\right) * \log (e)\left(-m_{k}-n_{k}-p_{k}\right) \\
& -\sum_{s=1}^{N} t_{k}\left(x_{s}, y_{s} ; \theta^{(l)}\right) \frac{1}{\Gamma m_{k}}\left(-\Gamma m_{k} * \log (\log (e))+\Gamma m_{k} * \psi\left(m_{k}\right) * \log (e)^{-m_{k}}\right)=0
\end{aligned}
$$

where, $\psi\left(m_{k}+n_{k}+p_{k}\right)=\operatorname{digamma}\left(m_{k}+n_{k}+p_{k}\right)$

The updated equation of $n_{k}$ at $(l+1)^{t h}$ iteration is 


$$
\begin{aligned}
& \sum_{s=1}^{N} t_{k}\left(x_{s,} y_{s} ; \theta^{(l)}\right) \log \left(y_{s}\right)+\sum_{s=1}^{N} t_{k}\left(x_{s,} y_{s} ; \theta^{(l)}\right) \frac{1}{\Gamma\left(m_{k}+n_{k}+p_{k}\right)}\left(-\Gamma\left(m_{k}+n_{k}+p_{k}\right)\right. \\
& \left.* \log (\log (e))+\Gamma\left(m_{k}+n_{k}+p_{k}\right) * \psi\left(m_{k}+n_{k}+p_{k}\right)\right) * \log (e)^{\left(-m_{k}-n_{k}-p_{k}\right)} \\
& -\sum_{s=1}^{N} t_{k}\left(x_{s}, y_{s} ; \theta^{(l)}\right) \frac{1}{\Gamma n_{k}}\left(-\Gamma n_{k} * \log (\log (e))+\Gamma n_{k} * \psi\left(n_{k}\right) * \log (e)^{-n_{k}}\right)=0
\end{aligned}
$$

where, $\psi\left(m_{k}+n_{k}+p_{k}\right)=\operatorname{digamma}\left(m_{k}+n_{k}+p_{k}\right)$

The updated equation of $p_{k}$ at $(l+1)^{t h}$ iteration is

$$
\begin{aligned}
& \sum_{s=1}^{N} t_{k}\left(x_{s,} y_{s} ; \theta^{(l)}\right) \log \left(1-x_{s}-y_{s}\right)+ \\
& \sum_{s=1}^{N} t_{k}\left(x_{s}, y_{s} ; \theta^{(l)}\right) \frac{1}{\Gamma\left(m_{k}+n_{k}+p_{k}\right)}\left(-\Gamma\left(m_{k}+n_{k}+p_{k}\right) * \log (\log (e))+\right. \\
& \left.\Gamma\left(m_{k}+n_{k}+p_{k}\right) * \psi\left(m_{k}+n_{k}+p_{k}\right)\right) * \log (e)^{\left(-m_{k}-n_{k}-p_{k}\right)}- \\
& \sum_{s=1}^{N} t_{k}\left(x_{s}, y_{s} ; \theta^{(l)}\right) \frac{1}{\Gamma\left(p_{k}\right)}\left(-\Gamma\left(p_{k}\right) * \log (\log (e))+\Gamma\left(p_{k}\right) * \psi\left(p_{k}\right) * \log (e)\right)^{-p_{k}}=0
\end{aligned}
$$

where, $\psi\left(m_{k}+n_{k}+p_{k}\right)=\operatorname{digamma}\left(m_{k}+n_{k}+p_{k}\right)$

Solving equations (7) (8) (9) and (10) iteratively using MATLAB code we get the revised estimates of $\alpha_{k}, m_{k}, n_{k,} p_{k}$ for $\mathrm{K}=1,2$.

\section{INITILIZATION OF MODEL PARAMETERS BY K-MEANS}

The efficiency of the EM-Algorithm in estimating the parameters is heavily dependent on the initial estimates of the parameters. The number of mixture components taken for K-means algorithm is two (skin and non-skin), i.e., $\mathrm{K}=2$. Usually the mixing parameter and the region parameters ( $\mathrm{m}, \mathrm{n}$, and $\mathrm{p}$ ) are unknown. A commonly used method in initialization is by drawing a random sample in the entire image data [32] [33]. This method perform well only when the sample size is large, and the computation time is heavily increased. When the sample size is small it is likely that some small regions may not be sampled. To overcome this problem, we use K-means algorithm [34] to divide the whole image into two homogeneous regions representing skin and nonskin regions. We obtain the initial estimates of the parameters $m_{k}, n_{k}$ and $p_{k}$ for each image region using the method of moment estimators for bivariate Pearson type II $a \alpha$ distribution and for the parameters $\alpha_{i}$ as $\alpha_{i}=\frac{1}{2}$ for $\mathrm{i}=1,2$.

Therefore the initial estimates of $m_{k}, n_{k}$ and $p_{k}$ can be obtained by equating the theoretical moments with the sample moments. This implies

$$
\begin{gathered}
\frac{m_{k}}{m_{k}+n_{k}+p_{k}}=\overline{x_{k}} \\
\frac{n_{k}}{m_{k}+n_{k}+p_{k}}=\overline{y_{k}}
\end{gathered}
$$

$$
\frac{-m_{k} n_{k}}{\left(m_{k}+n_{k}+p_{k}+1\right)\left(m_{k}+n_{k}+p_{k}\right)^{2}}=\overline{s_{x y k}}
$$

where, $\overline{x_{k}}$ is the $k^{\text {th }}$ region sample mean of the Hue.

$\overline{y_{k}}$ is the $k^{\text {th }}$ region sample mean of the Saturation.

$\overline{S_{x y k}}$ is the $k^{\text {th }}$ region sample between Hue and Saturation

Solving the equations (11), (12), (13) we get the initial estimates of the parameters $\left(m_{k}, n_{k}\right.$ and $\left.p_{k}\right)$ for $\mathrm{K}=1$, 2. Substituting these values as the initial estimates, we obtain the refined estimates of the parameters by using the EM-Algorithm.

\section{SKIN COLOUR SEGMENTATION ALGORITHM}

After refining the parameters the prime step is skin colour segmentation, by allocating the pixels to the skin or non-skin segments. This operation is performed by segmentation algorithm. The skin colour segmentation algorithm consists of the following steps

1. Divide the whole image into two regions using Kmeans algorithm

2. Obtain the initial estimates of the model parameters using the moment estimators as discussed in section 4 for each region

3. Obtain the refined estimates of the model parameters by using the EM-algorithm with the updated equations given in section 3 .

4. Substitute the estimated parameter values in the image joint probability density function

$h(x, y)=\sum_{i=1}^{K} \alpha_{i} f_{i}\left(x, y ; \theta_{i}\right)$

where $f_{i}\left(x, y / \theta_{i}\right)$ is as given equation (1).

5. Segment the pixels as skin colour or non-skin colour pixel using a threshold $(\mathrm{t})$ and the likelihood function such that $L(x / \theta) \geq t$ or $L(x / \theta)<t$ respectively for $0<\mathrm{t}<1$.

The optimal threshold value of $\mathrm{t}$ is determined computing true positive and false positive over the segmented regions and plotting the ROC Curve. 


\section{EXPERIMENTAL RESULTS AND PERFORMANCE EVALUATION}

In this section, the performance of the developed skin colour segmentation algorithm is evaluated. For this purpose the skin images are collected from JNTUK database. A random sample of 5 images is taken from the database and the feature vector consisting of hue and saturation for each pixel of the each image is computed utilizing HSI colour space. In HSI colour space the hue and saturation values are computed from the values of RGB for each pixel in the image using the formula

Hue $=\mathrm{H}=\cos ^{-1}\left[\frac{(R-G)+(R-B)}{2 \sqrt{(R-G)^{2}+(R-B)(G-B)}}\right], B \leq G$

Saturation $=\mathrm{S}=\frac{1-\min (R, G, B)}{I}$

where $\mathrm{I}=\frac{R+G+B}{3}$ is the intensity of pixel.

With the feature vector $(\mathrm{H}, \mathrm{S})$ each image is modeled by using the two component bivariate Pearson type II $a \alpha$ mixture distribution. The initial values of the model parameters are obtained by dividing all the pixels into two categories namely skin and non-skin region using $\mathrm{K}$-means algorithm with $\mathrm{K}=2$ and taking the moment estimates for $\left(m_{k}, n_{k}\right.$ and $\left.p_{k}\right), \mathrm{K}=1,2$. Using these initial estimates and the updated equations of the EM-Algorithm discussed in section.3 with MATLAB code the refined estimates of model parameters are obtained. Substituting the refined estimates in the bivariate Pearson type II $a \alpha$ joint probability distribution function the skin colour and non-skin colour models of each image are estimated. The segmentation algorithm with component maximum likelihood under Bayesian frame and a threshold value $\mathrm{t}$ as discussed in section 5 is used to segment the image. Figure. 1 shows the original and segmented images.

\begin{tabular}{|c|c|c|c|c|c|}
\hline Images & $\begin{array}{l}\text { Image 1 } \\
\text { (Male1) }\end{array}$ & $\begin{array}{c}\text { Image } 2 \\
\text { (Female1) }\end{array}$ & $\begin{array}{l}\text { Image } 3 \\
\text { (Male2) }\end{array}$ & $\begin{array}{c}\text { Image } 4 \\
\text { (Female2) }\end{array}$ & $\begin{array}{l}\text { Image } 5 \\
\text { (Male3) }\end{array}$ \\
\hline Original & & & & & \\
\hline Segmented & (8) & & A & & \\
\hline
\end{tabular}

Figure.1 Original and Segmented Images

The developed algorithm performance is evaluated by comparing skin colour segmentation algorithm with the Gaussian mixture model. Table.1 present the miss classification rate of the skin pixels of the sample image using proposed model and Gaussian mixture model.

Table.1 Miss Classification rate of the classifier

\begin{tabular}{|l|l|}
\hline Model & Miss Classification Rate \\
\hline BPT II $a \alpha$ MM & $3.5 \%$ \\
\hline GMM & $8.3 \%$ \\
\hline
\end{tabular}

From the Table.1 it is observed that the misclassification rate of the classifier with bivariate Pearson type II $a \alpha$ mixture model (BPTII $a \alpha \mathrm{MM}$ ) is less compared to that of GMM.

The accuracy of the classifier is also studied for the sample images by using confusion matrix for skin and non-skin regions. Table. 2 shows the values of TPR, FPR, Precision, Recall and F-measure for skin and nonskin segments of the sample images.

Table. 2 Comparative study of GMM and BPTII $a \alpha_{\mathrm{MM}}$

\begin{tabular}{|l|l|l|l|l|l|l|}
\hline Image & Method & TPR & FPR & Precision & Recall & F-measure \\
\hline \multirow{2}{*}{$\begin{array}{l}\text { Image1 } \\
\text { (Male1) }\end{array}$} & BPTII $a \alpha \mathrm{MM}$ & 0.9642 & 0.0625 & 0.9818 & 0.9642 & 0.9640 \\
\cline { 2 - 7 } & GMM & 0.9285 & 0.1875 & 0.9454 & 0.9285 & 0.9368 \\
\hline \multirow{2}{*}{$\begin{array}{l}\text { Image2 } \\
\text { (female1) }\end{array}$} & BPTII $a \alpha \mathrm{MM}$ & 0.9625 & 0.0181 & 0.9788 & 0.9625 & 0.9705 \\
\cline { 2 - 7 } & GMM & 0.9166 & 0.0833 & 0.9565 & 0.9166 & 0.9363 \\
\hline \multirow{2}{*}{$\begin{array}{l}\text { Image3 } \\
\text { (Male2) }\end{array}$} & BPTII $a \alpha \mathrm{MM}$ & 0.9692 & 0.0600 & 0.9767 & 0.9692 & 0.9729 \\
\cline { 2 - 7 } & GMM & 0.9307 & 0.0700 & 0.9718 & 0.9307 & 0.9508 \\
\hline \multirow{2}{*}{$\begin{array}{l}\text { Image4 } \\
\text { (female2) }\end{array}$} & BPTII $a \alpha \mathrm{MM}$ & 0.9629 & 0.0363 & 0.9848 & 0.9629 & 0.9737 \\
\cline { 2 - 7 } & GMM & 0.9259 & 0.0454 & 0.9803 & 0.9259 & 0.9260 \\
\hline \multirow{2}{*}{$\begin{array}{l}\text { Image 5 } \\
\text { (Male3) }\end{array}$} & BPTII $a \alpha \mathrm{MM}$ & 0.9800 & 0.0187 & 0.9849 & 0.9800 & 0.9824 \\
\cline { 2 - 7 } & GMM & 0.8750 & 0.0625 & 0.9459 & 0.8750 & 0.9090 \\
\hline
\end{tabular}

From Table. 2 it is obtained that the F-measure value for the proposed classifier is more. This indicates the proposed classifier perform better than that of Gaussian mixture model. Figure.2 shows the ROC curves associated with the proposed skin colour classifier and the classifier with GMM. 


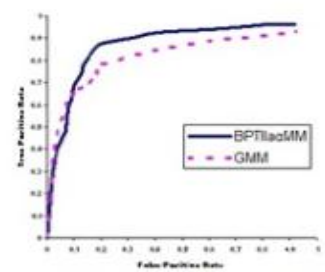

Image 1(Male1)

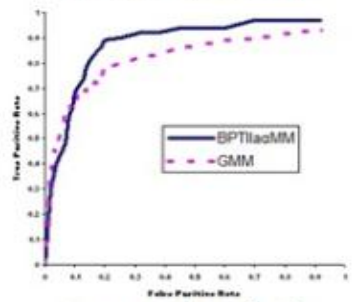

Image 3(Male2)

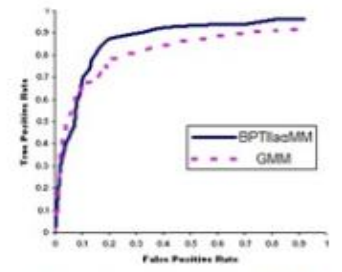

Image 2(Female1)

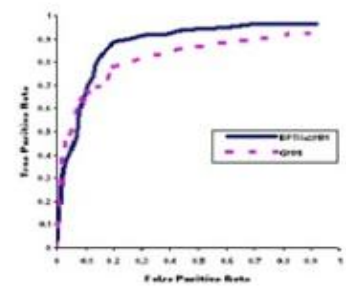

Image 4(Female2)

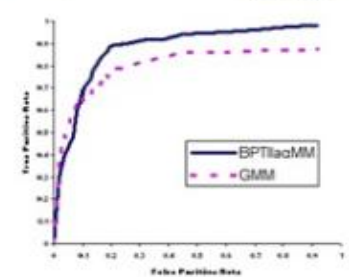

Image 5(Male3)

Figure. 2 ROC Curves

From Figure.2 it is observed that the proposed classifier is having less false detection of the skin pixels compared to the classifier with GMM. The figure also shows that, it can successfully identify the exposed skin region including face, hands and neck.

The performance of the segmentation algorithm is also studied by obtaining three segmentation performance measures namely, Probabilistic Rand Index (PRI) [35], Variation of Information (VOI) [36], Global Consistency Error (GCE) [37], with the sample images. The computed values of the performance measures for the developed algorithm with BPTII $a \alpha$ MM and GMM are presented in Table.3.

Table.3 Segmentation Performance Measures

\begin{tabular}{|c|c|c|c|c|}
\hline \multirow{2}{*}{ Image } & \multirow{2}{*}{ Method } & \multicolumn{3}{|c|}{ Performance Measures } \\
\hline & & PRI & GCE & VOI \\
\hline \multirow{2}{*}{$\begin{array}{l}\text { Image } 1 \\
\text { (Male1) }\end{array}$} & BPTII $a \alpha \mathrm{MM}$ & 0.6941 & 0.1928 & 0.0924 \\
\hline & GMM & 0.5128 & 0.2486 & 0.1529 \\
\hline \multirow{2}{*}{$\begin{array}{l}\text { Image 2 } \\
\text { (Female1) }\end{array}$} & BPTII $a \alpha \mathrm{MM}$ & 0.7810 & 0.2016 & 0.0816 \\
\hline & GMM & 0.5367 & 0.2249 & 0.0981 \\
\hline \multirow{2}{*}{$\begin{array}{l}\text { Image } 3 \\
\text { (Male2) }\end{array}$} & BPTII $a \alpha \mathrm{MM}$ & 0.6721 & 0.1281 & 0.0926 \\
\hline & GMM & 0.4826 & 0.1924 & 0.1626 \\
\hline \multirow{2}{*}{$\begin{array}{l}\text { Image } 4 \\
\text { (Female2) }\end{array}$} & BPTII $a \alpha \mathrm{MM}$ & 0.7941 & 0.0972 & 0.0831 \\
\hline & GMM & 0.6124 & 0.1362 & 0.1472 \\
\hline \multirow{2}{*}{$\begin{array}{l}\text { Image } 5 \\
\text { (Males) }\end{array}$} & BPTII $a \alpha \mathrm{MM}$ & 0.7214 & 0.1902 & 0.0662 \\
\hline & GMM & 0.5924 & 0.2136 & $0.12 / 1$ \\
\hline
\end{tabular}

From the Table.3 it is observed that the PRI value of the proposed algorithm for sample images considered for experimentation are more than that of the value from the segmented algorithm based on GMM and they are closed to 1. Similarly the GCE and VOI values of the proposed algorithm are less than that of finite Gaussian mixture model and close to 0 . This reveals that the proposed segmentation algorithm performs better than the algorithm with GMM and the proposed skin colour segmentation is close to the ground truth.

\section{CONCLUSION}

In this paper we proposed a skin colour segmentation algorithm based on bivariate Pearson type mixture model using HSI colour space. Here it is assumed that the bivariate feature vector (Hue and Saturation) of the whole image follows a bivariate Pearson type II $a \alpha$ mixture distribution which is capable of characterizing the skin and non-skin colours in the image. The bivariate Pearson type II $a \alpha$ distribution is having marginal distributions as beta distributions with range 0 and 1 , having the negative correlation coefficient and their features closely match the hue and saturation statistical characteristics of the skin and non-skin regions. This model also includes various types of bivariate distribution for specific values of the parameters. As this distribution is having three parameters only, it is a parsimonious model. The model parameters are obtained by deriving the updated equations of the EM-Algorithm. The initialization of the parameters is done using K-means algorithm and moment method of estimation. The experimental results with different types of five face images taken from JNTUK database revealed that the proposed algorithm perform much superior with respect to image segmentation performance metrics like PRI, GCE and VOI. A comparative study of proposed model with that of the GMM has shown that the proposed algorithm outperforms the existing algorithm for some images in skin colour segmentation. This is also supported by ROC curves. This algorithm can also be further extended to unsupervised skin colour segmentation with more number of regions of the image such as background, skin colour, non human objects etc. It is also possible to develop a skin colour segmentation based on bivariate Pearson type mixture model and hidden markov model which will be taken else where.

\section{REFERENCES}

[1] Alexander Wong, Jacob Scharcanski and Paul Fieguth (2011), "Automatic Skin Lesion Segmentation via Iterative Stochastic Region Merging”, IEEE Trans. on Information Technology in Biomedicine, Vol.15, No.6,pp.929-936.

[2] Wei Ren Tan, Chee Seng Chan, Pratheepan Yogarajah, and Joan Condell (2012), "A Fusion Approach for Efficient Human Skin Detection”, 
IEEE Transactions on Industrial Informatics, Vol.8, No.1,pp.138-147.

[3] Chang-Yul Kim, Oh-Jin Kwon, and Seokrim Choi (2011), "A Practical System for Detecting Obscene Videos",IEEE Transactions on Consumer Electronics, Vol.57, No.2, pp. 646-650.

[4] Fumihito Yasuma, Tomoo Mitsunaga, Daisuke Iso, and Shree K. Nayar (2010), "Generalized Assorted Pixel Camera: Postcapture Control of Resolution, Dynamic Range and Spectrum", IEEE Transactions on Image Processing, Vol.19, No.9, pp.2241-2253.

[5] C. Chen, S.P. Chiang (1997), "Detection of human faces in colour images", IEE Proc. Vision Image Signal Process, Vol.144 (6) pp.384-388.

[6] H. Wu, Q. Chen, M. Yachida (1999), "Face detection from color images using a fuzzy pattern matching method", IEEE Trans. Pattern Anal.Mach. Intell. Vol. 21 (6) pp.557-563.

[7] M.H. Yang, N. Ahuja (1999), “Gaussian Mixture model for human skin color and its application in image and video databases", Proceedings of SPIE: Conference on Storage and Retrieval for Image and Video Databases, Vol. 3656, pp. 458-466.

[8] L.M. Bergasa, M. Mazo, A. Gardel, M.A. Sotelo, L. Boquete (2000), "Unsupervised and adaptive Gaussian skin-color model", Image Vision Comput. Vol.18 (12) pp.987-1003.

[9] D. Brown, I. Craw, J. Lewthwaite (2001), “A SOM based approach to skin detection with application in real time systems", BMVC01.

[10] S. McKenna, S. Gong, Y. Raja (1998), "Modeling facial colour and identity with Gaussian mixtures", Pattern Recognition Vol.31 (12) pp.1883-1892.

[11] J.C. Terillon, M. David, S. Akamatsu (1998), "Detection of human faces in complex scene images by use of a skin color model and of invariant Fourier-Mellinmoments", ICPR98, pp. $350-1355$.

[12] C. Garcia, G. Tziritas (1999), "Face detection using quantized skin color regions merging and wavelet packet analysis”, IEEE Trans. Multimedia Vol.1 (3) pp. 264-277.

[13] Y. Wang, B. Yuan (2001), "A novel approach for human face detection from color images under complex background", Pattern Recognition Vol.34 (10) pp.1983-1992.

[14] Q. Zhu, K.-T. Cheng, C.-T. Wu, Y.-L. Wu (2004), "Adaptive learning of an accurate skin-color model", AFGR04.

[15] Y. Dai, Y. Nakano (1996), "Face-texture model based on SGLD and its application in face detection in a color scene", Pattern Recognition Vol. 29 (6) pp.1007-1017.
[16] D. Chai, K.N. Ngan (1998), "Locating facial region of a head-and-shoulders color image", ICFGR98.

[17] D. Chai, A. Bouzerdoum (2000), “A Bayesian approach to skin color classification in $\mathrm{YCbCr}$ color space", IEEE TENCON00, Vol. 2 pp. 421424.

[18] F. Marques, V. Vilaplana (2000), “A morphological approach for segmentation and tracking of human face", ICPR 2000.

[19] G. Gomez, M. Sanchez, L.E. Sucar (2002), “On selecting an appropriate colour space for skin detection", Springer-Verlag: Lecture Notes in Artificial Intelligence, Vol. 2313, pp. 70-79.

[20] J.J. de Dios, N. Garcia, (2003), "Face detection based on a new color space YCgCr", ICIP03.

[21] S. Kawato, J. Ohya,( 2000), “Automatic skin-color distribution extraction for face detection and tracking", Fifth International Conference on Signal Processing, Vol. 2 pp. 1415-1418.

[22] G. Wyszecki, W.S. Stiles (1967), Color Science, Wiley, New York.

[23] G.V.S. Raj Kumar, K.Srinivasa Rao and P.Srinivasa Rao (2011), "Image Segmentation and Retrievals based on finite doubly truncated bivariate Gaussian mixture model and K-means", International Journal of Computer Applications, Vol. 25. No.5 pp.5-13.

[24] Rafel C Gonzalez and Richard E Woods (2001), "Digital Image Processing", Pearson education, India

[25] K.Srinivasa Rao, B.N.Jagadesh, Ch.Satyanarayana (2012), "Skin Colour Segmentation using Fintte Bivariate Pearsonian Type-IVa Mixture Model", Computer Engineering and Intelligent Systems, Vol.3, No.5, pp.46-55.

[26] J. Cai, A. Goshtasby (1999), "Detecting human faces in color images", Image and Vision Computing 18, pp.63-75.

[27] Hayit Greenspan, Jacob Goldberger, Itay Eshet (2001) "Mixture model for face color modeling and segmentation" Pattern Recognition Letters 22 (2001), pp.1525-1536.

[28] P.Kakumanu, S.Makrogiannis, N. Bourbakis (2007) "A survey of skin-color modeling and detection methods", Pattern Recognition, vol.40, pp.11061122,20

[29] Ki-Won Byun and Ki-Gon Nam(2012), "Skin Region Detection Using a Mean Shift Algorithm Based on the Histogram Approximation",Vol.13,No.1,pp.10-15.

[30] Jianyong Sun , Ata Kabán b, Jonathan M. Garibaldi(2010), "Robust mixture clustering using 
Pearson type VII distribution”, Pattern Recognition letters.

[31] Norrman L. Johnson, Samuel Kotz and Balakrishnan (2000), "Continuous Multivariate Distributions", John Wiley and Sons Publications, New York.

[32] Mclanchlan G. and Krishnan T. (1997), "The EM Algorithm and Extensions", John Wiley and Sons, New York -1997.

[33] Mclanchlan G. and Peel D.(2000) "The EM Algorithm For Parameter Estimations”, John Wiley and Sons, New York.

[34] Rose H.Turi (2001), Cluster Based Image Segmentation, PhD Thesis, Monash University, Australia

[35] Unnikrishnan R., Pantofaru C., and Hernbert M. (2007), "Toward objective evaluation of image segmentation algorithms", IEEE Trans.Pattern Annl.Mach.Intell, Vol.29 ,No.6, pp. 929-944.

[36] Meila M. (2005) Comparing Clustering - An axiomatic view, in proc.22nd Int. Conf. Machine Learning, pp. 577-584.

[37] Martin D. Fowlkes C., Tal D. and Malik J. (2001) A database of human segmented natural images and its application to evaluating segmentation algorithms and measuring ecological statistics, in proc. 8th Int. Conference Computer vision, Vol.2 pp.416- 423.

\section{AUTHOR'S PROFILE}

Mr.B.N.Jagadesh is presently working as Assistant Professor in Computer science and Engineering Department at Srinivasa Institute of Engineering \& Technology, Cheyyeru. He presented several research papers in national and International conferences and seminars. He published a good number of papers in national and International journals. He guided several students for getting their M.Tech degrees in Computer Science \& Engineering. His current research interests are Image Processing, Speech Processing and Data Mining.

Dr. K.Srinivasa Rao is presently working as Professor, Department of Statistics, Andhra University, Visakhapatnam. He is elected chief editor of Journal of ISPS and elected Vice-President of Operation Research of India. He guided 28 students for Ph.D in Statistics, Mathematics, Computer Science, Electronics and Communications Engineering, Industrial Engineering and Operations Research. He published 102 research papers in national and International journals with high reputation. His research interests are Image Processing, Communication Systems, Data Mining and stochastic models.
Dr. Ch. Satyanarayana is Associate Professor in Computer science and Engineering Department at Jawaharlal Nehru Technological University Kakinada, Kakinada. He has 13 years of experience. His area of interest is on Image processing, Database Management Systems, Speech Recognition, Pattern recognition and network security. He published more than 20 research papers in international journals. He published more than 30 research papers in international conferences. 